\title{
An Epidemic of Scurvy, Identified Based on Lower Extremity Swelling, in a Southern Ethiopian Prison
}

\author{
Wondwossen Amogne,${ }^{1 *}$ Meskerem Nimani, ${ }^{2}$ Ishmael Shemsedin, ${ }^{2}$ Wadu Marshalo, ${ }^{1}$ Daddi Jima, ${ }^{3}$ Adamu Addissie,,${ }^{1}$ and \\ John Fogarty ${ }^{4}$ \\ ${ }^{1}$ Addis Ababa University, College of Health Sciences, Addis Ababa, Ethiopia; ${ }^{2}$ Addis Ababa Emergency Burn \& Trauma Hospital, Addis Ababa, \\ Ethiopia; ${ }^{3}$ Ethiopian Public Health Institute, Addis Ababa, Ethiopia; ${ }^{4}$ Department of Family \& Community Medicine, University of New Mexico, \\ Albuquerque, New Mexico
}

\begin{abstract}
In October 2016, we received reports of five deaths among prisoners with leg swelling of unknown etiology in southwestern Ethiopia. A descriptive cross-sectional study was conducted to investigate the outbreak. A suspected case was defined as a prisoner with leg swelling of unknown etiology noted between May 15, 2016 and November 29, 2016. A total of 118 suspected cases were identified with unilateral or bilateral leg swelling without an identifiable cause from a total of 2,790 prisoners. Eight of the suspected cases were thoroughly examined, and seven of these suspected cases had clinical findings consistent with scurvy. Three of the clinical cases had undetectable vitamin $\mathrm{C}$ levels in the serum. The attack rate for the prison was $4.2 \%(118 / 2,790)$, and 11 death s were identified, making the case fatality rate $9.3 \%$ (11/118). Clinical cases of scurvy had symptoms of fatigue, myalgia, arthralgia, and signs of follicular hyperkeratosis, petechiae, peripheral edema, and oral lesions. All clinical cases had severe anemia with hemoglobin $<6.0 \mathrm{~g} / \mathrm{dL}$. The diet provided by the prison excluded fruits and vegetables. Scurvy was determined to be the cause of the outbreak, and vitamin $\mathrm{C}$ supplementation was promptly initiated. All symptomatic prisoners improved, and no further cases were identified in a 4-week follow-up period of active surveillance.
\end{abstract}

\section{INTRODUCTION}

Scurvy is one of the oldest human diseases. Descriptions of scurvy have been found in the Old Testament, in the writings of Pliny, and in Egyptian medical scrolls from 1500 B.C. ${ }^{1,2}$ Archeological evidence of scurvy has been found across the globe from Asia, Europe, Africa, and North America. ${ }^{3-6}$

The disease is caused by a deficiency of vitamin $\mathrm{C}$ (ascorbic acid), which is an electron donor and reducing agent. Vitamin $\mathrm{C}$ is critical to the functioning of 15 mammalian enzymes, including three being important to collagen synthesis. ${ }^{7}$

Humans, along with monkeys and guinea pigs, are among the few species unable to synthesize vitamin C. Stores of vitamin $\mathrm{C}$ also are limited in humans, and clinical disease becomes evident within 2-4 months of inadequate intake. ${ }^{8-10}$

Scurvy characteristically has been associated with extended expeditions at sea and has previously caused a large burden of disease among sailors and explorers. It is estimated that two million sailors died of scurvy from the 16th through the 19th centuries. ${ }^{11}$ "Land scurvy" also has affected vulnerable populations on land during times of war and famine, such as during the American Civil War and the Irish potato famine. ${ }^{12,13}$

With the finding that citrus fruits could prevent scurvy in 1747 followed by British Navy recommendations to add lemon juice to voyages, the global incidence of scurvy declined through the 19th century. ${ }^{14}$ After the discovery of vitamin C in $1928,{ }^{15}$ scurvy became a relatively rare disease, and few practicing physicians today have encountered a patient with scurvy.

Nonetheless, given the inability to synthesize vitamin C, humans remain at risk for scurvy when diets are limited. The medical literature is replete with case reports from many continents describing isolated cases of scurvy associated with chronic disease, alcohol dependence, mental illness, or social disruption. ${ }^{16-20}$

*Address correspondence to Wondwossen Amogne, Addis Ababa University, College of Health Sciences, Addis Ababa, Ethiopia. E-mail: wondwossen.amogne@aau.edu.et
Large outbreaks of scurvy still occur, especially among vulnerable populations such as refugees and prisoners. It is estimated that scurvy affected more than 100,000 refugees in Somali and Sudanese camps during the 1980s. Outbreaks of scurvy in the East African camps typically peaked about 4 months after consuming a diet of vitamin C-deficient food. ${ }^{21}$

Because of the rarity and unfamiliarity of scurvy among clinicians and public health workers, there can be a delay in diagnosis and treatment. ${ }^{22-24}$ For example, in 2002 an epidemic of scurvy in rural Afghanistan was initially misdiagnosed as an outbreak of Crimean-Congo hemorrhagic fever. Aid workers were evacuated, and intervention was delayed until a WHO investigation determined the cause to be scurvy. ${ }^{25,26}$

Scurvy was identified in 38 patients from a prison in southern Ethiopia in 2010. All 38 patients had been referred to a local hospital for evaluation of leg swelling and found clinically to have scurvy. Prisoners who had been incarcerated for $>8$ months were at increased risk of developing symptomatic scurvy. In addition to leg swelling, these patients frequently were noted to have cough, gum hypertrophy, hyperpigmentation, and anemia. ${ }^{27}$

On October 24, 2016, we received a report of an outbreak of leg swelling that included five deaths among prisoners at a prison in southern Ethiopia. We investigated to describe the magnitude of the outbreak, identify risk factors and etiology, and propose control measures.

\section{BACKGROUND AND METHODS}

The prison is located in southwestern Ethiopia in the Southern Nations, Nationalities, and Peoples' Regional State (SNNPR) near the border with South Sudan. The area is considered temperate and tropical, and subsistence farming is the main industry. Coffee, spices, and fruits, including pineapple, oranges, and bananas, are the principal cash crops.

At the time of the investigation, the prison population included 2,677 adult men, 88 adult women, and 25 children. Incarcerated mothers were allowed to keep their young children with them in a separate ward. The prison clinic was staffed by four nurses, one laboratory technician, and one 
health officer. The town where the prison is located also has a hospital staffed by general practitioners and an internal medicine specialist who can evaluate referrals from the prison.

Descriptive epidemiology. From October 25, 2016 to November 20, 2016, we investigated to identify suspected cases with leg swelling and determine the etiology of the outbreak and risk factors associated with the disease. We reviewed the prison clinic and local hospital medical records during the investigation period and for 5 years before the investigation. We also used epidemic line lists from the local health department.

We defined a suspected case as a prisoner at the prison with either unilateral or bilateral leg swelling who did not have a known explanation for the swelling. We included patients who had reported symptoms of ongoing leg swelling that had started after May 15, 2016 because we noted there had been a marked increase in leg swelling reported on epidemic line lists during that week.

Eight suspect cases with leg swelling were transferred for specialized medical evaluations in Addis Ababa to develop a broader differential diagnosis and ascertain a clinical diagnosis if possible. On October 25-26, 2016, eight patients had independent physical examinations performed by four senior clinicians (WA, JF, IS, MN). On-site clinical examination of additional patients at the prison in Southern Nations, Nationalities, and Peoples' Regional State by senior clinicians was not logistically possible.

We could not identify an international standard case definition for scurvy and therefore used descriptions of patients with experimentally induced scurvy to define a clinically confirmed case of scurvy as a prisoner with leg swelling plus petechial hemorrhages and/or hyperkeratotic follicular papules. Petechiae are known to be an early sign of scurvy, and hyperkeratosis is a specific sign, having been found in all experimentally induced scurvy patients. ${ }^{4}$ We defined a laboratory-confirmed case as a clinically confirmed case who had vitamin $\mathrm{C}$ levels below a reference of $0.8 \mathrm{mg} / \mathrm{L} .{ }^{29}$

Laboratory investigations. Blood samples were obtained from three patients with clinically confirmed scurvy who had been examined in Addis Ababa. After consent, 5-mL blood samples were taken, added to lithium heparin tubes, and centrifuged. The supernatant heparin plasma was transferred to new tubes and then immediately frozen. The three frozen

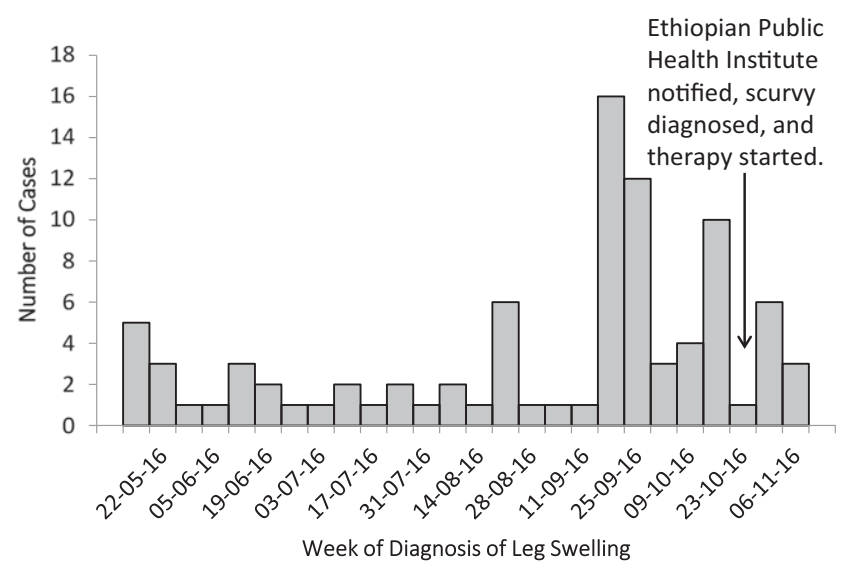

FIGURE 1. The number of suspect cases of scurvy by the week of diagnosis of leg swelling of unclear etiology, Southern Nations, Nationalities, and Peoples' Regional State, Ethiopia, 2016. samples were transferred by air to Bioscientia Institute for Medical Diagnostics, Ingelheim, Germany. Highperformance liquid chromatography analysis for vitamin $\mathrm{C} \mathrm{lev-}$ els was performed.

Environmental investigations. We evaluated living quarters and conditions, and we investigated the diet, water systems, and water sources in the prison. We also conducted key informant open-ended interviews with regional and zonal surveillance officers and prison staff.

\section{RESULTS}

Descriptive epidemiology. We reviewed prison medical records for the last 5 years and determined that the baseline number of cases of leg swelling was between two and eight cases per year. We noted an increase in the number of reported cases of leg swelling (i.e., six cases in 1 week) during the week of May 15, 2016 (Figure 1).

During the week of September 19, 2016, 15 prisoners were diagnosed with leg swelling by the prison clinic. The Regional Health Department and the Ethiopia Public Health Institute were notified on October 24, 2016, and an investigation was initiated the following day.

We identified 118 suspected cases between May 15, 2016 and November 3, 2016. Seven of eight suspected cases who received comprehensive clinical examinations were determined to have clinically confirmed scurvy. Three of the clinically confirmed cases had laboratory testing for vitamin C levels, and all three had nondetectable levels of vitamin C (laboratory-confirmed scurvy).

TABLE 1

Percentage of clinically confirmed scurvy patients $(N=7)$ with relevant symptoms of scurvy, Southern Nations, Nationalities, and Peoples' Regional State, Ethiopia, October 2016

\begin{tabular}{lr}
\hline \multicolumn{1}{c}{ Patient characteristics } & Value \\
\hline Mean age, years & 24.2 \\
Male sex, \% & 100 \\
Symptoms, \% & \\
Bruising & 86 \\
Arthralgia & 100 \\
Myalgia & 100 \\
Fatigue & 100 \\
Bloody diarrhea & 71 \\
Orthopedic findings, \% & \\
Ankle arthritis* & 57 \\
Knee arthritis* & 100 \\
Muscle tenderness with palpation & 100 \\
Inability to walk without assistance & 86 \\
Unilateral leg edema & 43 \\
Bilateral leg edema & 57 \\
Dermatologic findings, \% & 100 \\
Hyperpigmentation & 100 \\
Diffuse petechiae & 86 \\
Hyperkeratotic follicular papules & \\
Oral findings, \% & 57 \\
Halitosis & 71 \\
Bleeding gums & 71 \\
Loosening of teeth & \\
Laboratory findings, \% & 71 \\
Microscopic hematuria & 71 \\
Hypoalbuminemia ( $<3$ g/dL) & $100 \dagger$ \\
Anemia (Hgb < 6 g/dL) & \\
Leukopenia ( $<3,000$ cells/mm $\left.{ }^{3}\right)$ & \\
Serum vitamin C level $(<0.8$ mg/L) & \\
\hline *Clinically confirmed arthritis. & \\
tSerum vitamin C levels were available for only three patients. & \\
\end{tabular}




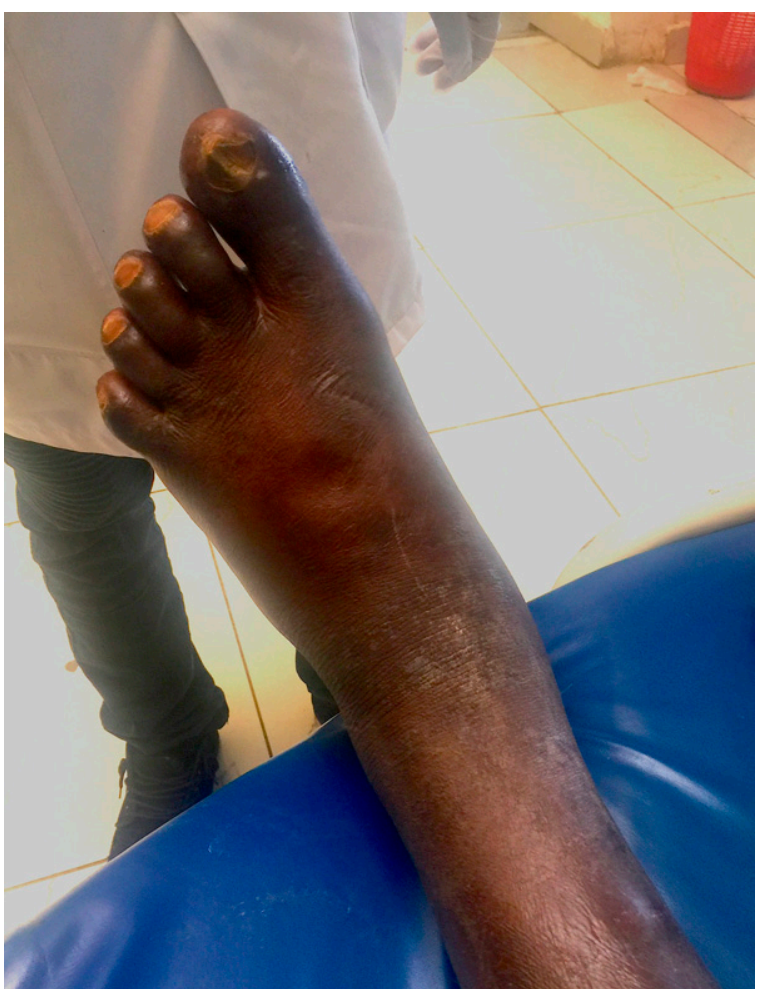

FiguRE 2. Pitting edema in a patient with laboratory-confirmed scurvy, Southern Nations, Nationalities, and Peoples' Regional State, Ethiopia, October 2016. This figure appears in color at www.ajtmh.org.

Eleven of the 118 suspect cases died, and 8 of the 11 died between October 16 and 29. The exact cause of death was not determined by autopsy. There were no fatalities among the clinically confirmed cases. All suspect cases were male, and the mean age was 34 years. No women or children were identified with symptoms of scurvy. The attack rate for the prison was $4.0 \%$, and the case fatality rate was $9.5 \%$.

Prisoners with leg swelling of unclear etiology previously had been evaluated by the prison health officer and physicians at the local hospital and had been assigned preliminary diagnoses before a definitive diagnosis of scurvy being made in Addis Ababa. The initial diagnoses most commonly made were deep venous thrombosis, arthritis, cellulitis, and sexually transmitted infections.

Clinical findings. Eight suspect cases were transferred from the prison to Addis Ababa for evaluation by senior clinicians. Seven of eight of the suspect cases examined in Addis Ababa met clinical criteria for scurvy, and the clinical findings from these patients are reported in Table 1. The suspect case who did not meet the criteria for scurvy had unilateral leg swelling from a knee injury.

Signs and symptoms. All seven clinically confirmed cases had symptoms of myalgias, arthralgias, fatigue, and unquantified weight loss. Five of seven had a history of bloody diarrhea.

All seven clinically confirmed case-patients had either unilateral or bilateral leg pitting edema (Figure 2). Six of seven were unable to walk without the use of a cane or support, and one was unable to sit upright or rise from the bed without complete assistance (Figures 3 and 4).

All seven clinically confirmed cases had findings of petechiae of varying sizes, mostly over the legs and feet

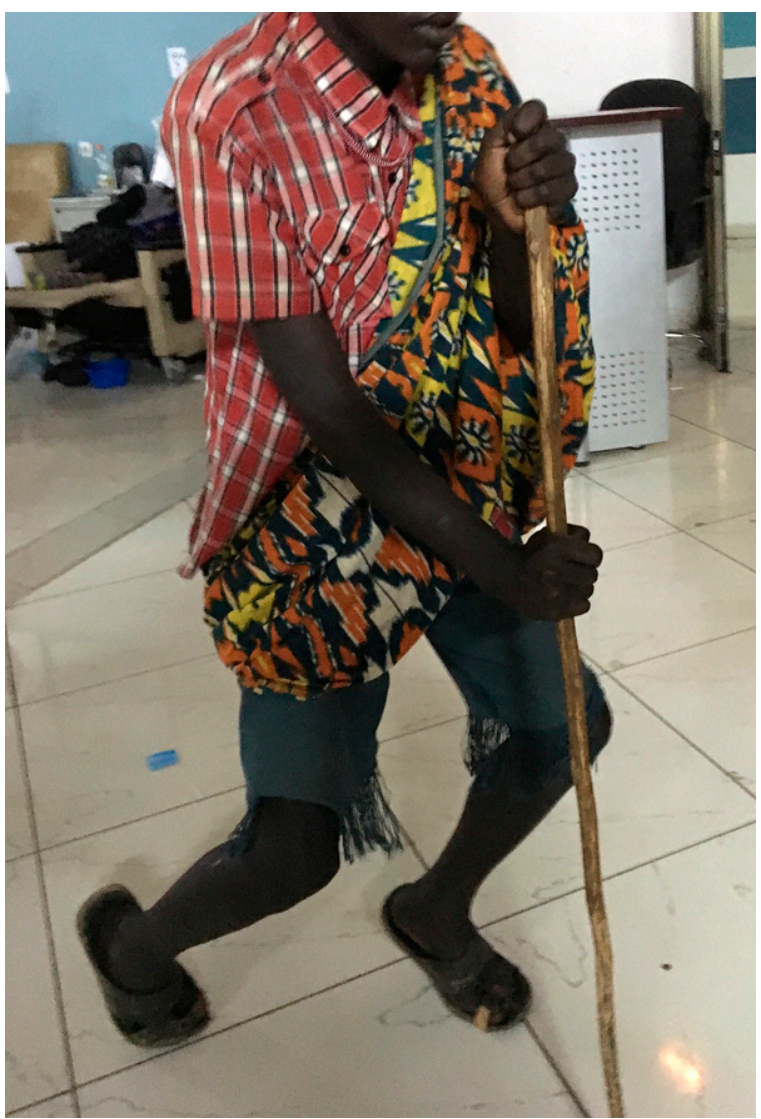

FIGURE 3. A patient with laboratory-confirmed scurvy and difficulty walking secondary to a severely restricted range of motion of the knees, Southern Nations, Nationalities, and Peoples' Regional State, Ethiopia, October 2016. This figure appears in color at www.ajtmh.org.

(Figure 5), and six had hyperkeratotic follicular papules (Figure $6)$. Five of seven had gum disease and/or severe halitosis.

Laboratory findings. Severe anemia $(\mathrm{Hb}<6.0 \mathrm{~g} / \mathrm{dL})$ was found in all seven clinically confirmed case-patients. The anemia was characterized as microcytic hypochromic in five of the cases; two cases had normochromic normocytic

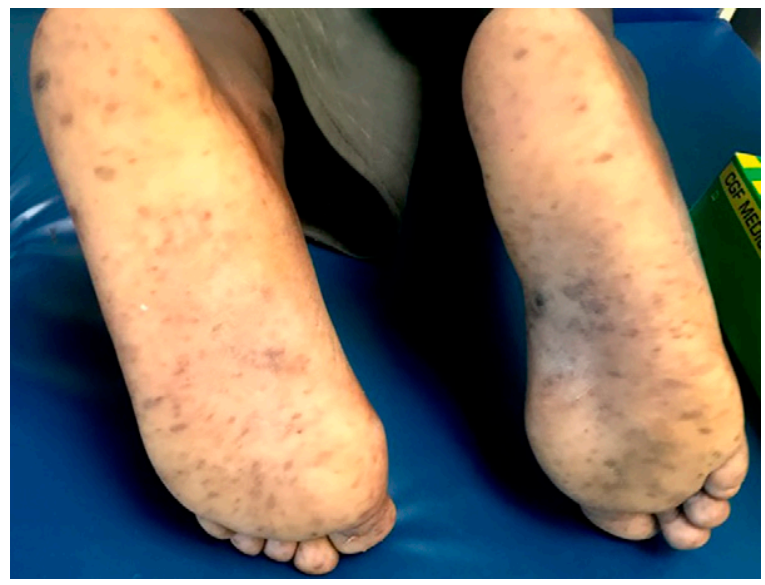

FIGURE 4. Petechiae over the feet of a patient with laboratoryconfirmed scurvy, Southern Nations, Nationalities, and Peoples' Regional State, Ethiopia, October 2016. This figure appears in color at www.ajtmh.org. 
features. Hypoalbuminemia (albumin $<3.0 \mathrm{~g} / \mathrm{dL}$ ), leukopenia (WBC $<2,800 / \mu \mathrm{L})$, and microscopic hematuria were found in five of seven clinical confirmed case-patients. All three samples from clinically confirmed cases showed nondetectable levels of vitamin C.

Environmental investigations. At the time of the investigation, there were 2,790 prisoners held in 18 rooms. The diet at the prison consisted almost entirely of cereals. Before March 2016 fruits and vegetables had been supplied to all prisoners at least twice weekly. However, in March 2016 a large outbreak of acute watery diarrhea (AWD) affected many communities in the southern part of Ethiopia. To protect against an AWD outbreak in the prison, the provision of fruits and vegetables by the prison food service was suspended. Some prisoners, especially women and children, maintained access to fruits and vegetables sporadically when family members delivered them for personal consumption. Our investigation revealed that many prisoners did not have access to fruits or vegetables from mid-March 2016 to October 27, 2016.

Treatment and public health actions. A clinical diagnosis of scurvy as the cause of the outbreak was made on October 26,2016 . Vitamin C supplementation was initiated immediately, and prisoners began receiving ascorbic acid $500 \mathrm{mg}$ orally daily. Additionally, the diet for prisoners was expanded to include fresh citrus fruits daily. Supplemental $\mathrm{FeSO}_{4}$ tablets (200 mg; $65 \mathrm{mg}$ of elemental Fe) three times daily were provided.

The final suspected case was diagnosed with leg swelling on November 3, 2016. There were no additional prisoners diagnosed with scurvy and no deaths attributable to scurvy during 4 weeks of active surveillance following the outbreak investigation.

\section{DISCUSSION}

We identified an outbreak of scurvy in a prison attributed to a change in diet that contained no substantial sources of vitamin C. Fruits and vegetables had been mostly removed from the diet at the prison during an outbreak of AWD that was occurring in southern Ethiopia. The diet for many male prisoners mostly consisted of grains lacking vitamin $\mathrm{C}$ for approximately 6 months before our investigation. Women and children maintained access to food from outside the prison, and we did not identify any cases among those populations.

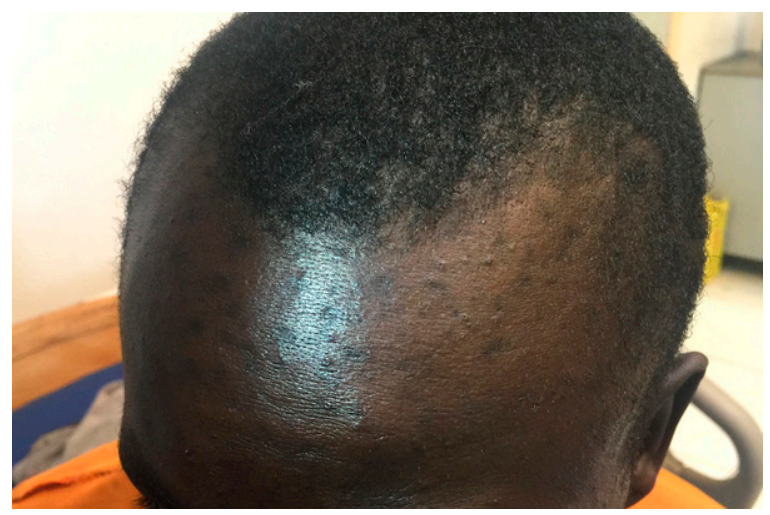

FIGURE 5. Perifollicular hemorrhagic papules in a patient with laboratory-confirmed scurvy, Southern Nations, Nationalities, and Peoples' Regional State, Ethiopia, October 2016. This figure appears in color at www.ajtmh.org.
Given our narrow case definition for suspected cases, it is likely that there were more prisoners with scurvy than we identified as suspect cases. Consequently, our reported attack rate of $4.2 \%$ is likely an underestimate, and the case fatality rate of $9.3 \%$ is an overestimate.

The number of deaths associated with this outbreak underscores the importance of early diagnosis and treatment of scurvy. Because scurvy is rare in contemporary society, most clinicians and public health workers remain unfamiliar with the signs and symptoms of the disease. The medical literature has for decades contained reports describing the difficulties in diagnosing scurvy and has offered warnings about delays in diagnosis. ${ }^{19,20,25-27,30,32}$

Patients described in case reports in the literature are frequently misdiagnosed with rheumatologic, autoimmune or bone diseases, deep venous thrombosis, or infectious diseases such as meningococcus or syphilis. ${ }^{14}$ The patients with scurvy in our report were most commonly misdiagnosed with deep venous thrombosis, arthritis, or sexually transmitted infections. Misdiagnoses of scurvy epidemics can result in delays in life-saving treatment.

Clinical findings of scurvy. The clinical findings in our report are consistent with findings in patients with experimentally induced scurvy. In studies of prisoners in an lowa state penitentiary who were deprived of all vitamin $\mathrm{C}$, the chief symptoms were fatigue, myalgias, arthralgias followed by more specific signs of follicular hyperkeratosis, petechial hemorrhage and peripheral edema, and swollen gums. Dermatologic changes occurred $8-13$ weeks after vitamin $C$ restriction, and oral lesions began after 11-19 weeks. ${ }^{8,28}$

In another study conducted during World War II, volunteers in England were fed a diet with restricted vitamin $\mathrm{C}$ content (10-70 mg/day). Within 17-21 weeks, subjects developed characteristic hyperkeratotic papules, and after 30-36 weeks they developed purple, swollen, bleeding gums. In another case study of experimentally induced scurvy, a physician restricted all vitamin $\mathrm{C}$ intake. He developed fatigue at 12 weeks, hyperkeratotic lesions at 19 weeks, petechiae at 23 weeks, and poorly healing wounds at 26 weeks. ${ }^{2}$

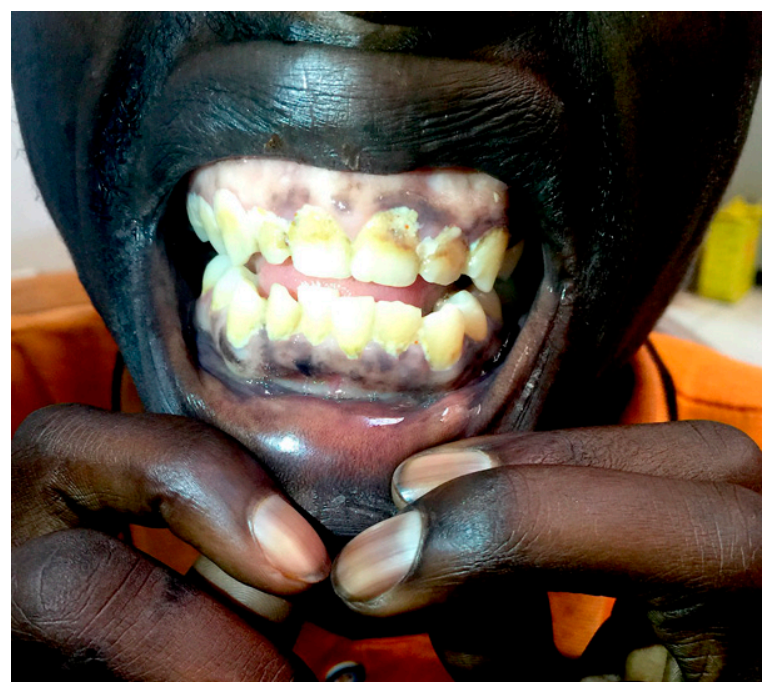

FIGURE 6. Gum disease in a patient with laboratory-confirmed scurvy, Southern Nations, Nationalities, and Peoples' Regional State, Ethiopia, October 2016. This figure appears in color at www.ajtmh.org. 
As can be seen from these reports of experimental scurvy, the clinical disease has a typical progression. The initial findings after restriction of vitamin $C$ intake include fatigue and lassitude followed by myalgias and joint pains. These symptoms are followed by clinical signs of edema, arthritis, rash, and oral lesions. The rapidity of onset of symptoms appears dependent upon the degree of restriction of vitamin C.

Perhaps most striking in our examination of clinically confirmed cases was that six of seven were unable to walk without the use of a cane or support, and one was completely bedridden. The patients were unable to passively or actively extend fully their knees and elbows, giving the clinical appearance of "flexion contractures" (Figure 4). Joint and musculoskeletal pains along with leg swelling are known to be common in scurvy and are accompanied by bleeding into the muscles and soft tissues. ${ }^{33-35}$ The swelling noted in the seven clinical confirmed cases in this report was thought to be multifactorial, arising from hypoalbuminemia as well as subcutaneous and fascial hemorrhages as described in prior reviews. ${ }^{36}$

Some of the more specific findings of scurvy are dermatologic and include follicular hyperkeratosis, ecchymoses, petechiae, and perifollicular hemorrhages. ${ }^{31}$ All clinically confirmed cases in our report had evidence of dermatologic changes (Figures 4 and 5).

Scurvy is known among laypersons as being associated with loss of teeth and gum abnormalities. These oral findings can include a purplish discoloration of the gums, hypertrophy, friability, and eventually tooth loss. ${ }^{32,37}$ All clinically confirmed cases in our review had notable halitosis and/or identifiable changes of gums, including the loss of teeth (Figure 6).

The patients in our report who were identified with clinical confirmed and laboratory-confirmed scurvy had classic clinical findings. Their signs and symptoms were nearly identical to a description of scurvy in 1753 by a British physician, James Lind.

Dr. Lind was a naval officer who conducted the first clinical trial showing that citrus fruits could prevent scurvy among sailors. In our review, we could find no better or more succinct description of scurvy than that provided by Dr. Lind more than two centuries ago. In his words: "In the early stages, patients do not look very sick, but are listless, perhaps a little pale, and have an aversion to exercise ... in a few days develop universal lassitude, stiffness, and feebleness of the knees, some difficulty breathing, itchy gums, and rough, dry skin splattered with bluish and reddish spots. These hemorrhages vary from the size of lentil to a handbreadth, mostly on the legs, less on the arms and trunk, and rarely on the head or face. The gums, however, bleed readily and become first soft and spongy and then putrid and rotten. The breath is foul, and the teeth fall out. The legs swell, at first only in the evening, but later all the time. Ulcers appear on the thighs, coated with blood and gore, and will not heal. Broken bones will not mend and even old healed wounds may break apart. The patients are now susceptible to whatever infection is going around, often diarrhea. Many joints, but especially the knees, are very painful and greatly swollen with blood. Patients now have abdominal pains cannot eat, are jaundiced and swollen all over, breathe with pain and labor, and may die suddenly."38

Limitations. The exact number of persons affected by vitamin $C$ deficiency in this outbreak is not known because we relied on a narrow case definition for suspect cases (leg swelling) and used primarily prison and local health records to identify suspect cases. In a study of experimentally induced scurvy, only three of five patients developed edema. ${ }^{8}$ Consequently, we believe the size of this outbreak was larger than what we have described.

Logistically it was not possible to conduct expert clinical examinations on all prisoners. Persons who had only subtle or early findings of scurvy, such as fatigue or dermatologic changes, would not have been included as cases. We also could only infer from the health records that prisoners who died of an unknown cause and also had leg swelling documented during the period of the investigation had died of scurvy.

\section{CONCLUSION}

We identified a large outbreak of scurvy in a prison that included 11 deaths. Health professionals working with vulnerable populations should maintain an index of suspicion using broader differential diagnoses that include scurvy. They also should be educated on the signs and symptoms of the disease. Surveillance systems that can detect diseases associated with micronutrient deficiencies such as vitamin $\mathrm{C}$ should be established for high-risk populations. ${ }^{39}$ The experiences described in this report and previous outbreaks of scurvy among prisoners and refugees in Afghanistan, Somalia, Sudan, and Ethiopia underscore the importance of having such surveillance systems in place. ${ }^{21,25,27,40}$

Received September 23, 2020. Accepted for publication April 12, 2021.

Published online June 28, 2021.

Acknowledgments: We thank the Southern Nations, Nationalities and Peoples Regional Health Bureau, Public Health Emergency Management, and Prison Clinic; Benchi Maji Zone Administration and Health Department; the Mizan Prison Institute; Addis Ababa Emergency Burn \& Trauma Hospital; the School of Public Health at the Addis Ababa University College of Heath Sciences; and the Ethiopian Public Health Institute for their assistance in investigating the outbreak and caring for the patients. The American Society of Tropical Medicine and Hygiene (ASTMH) assisted with publication expenses.

Disclosure: The Ministry of Health of Ethiopia gave the directive and approval to investigate this outbreak. In agreement with the International Ethical Guidelines for Epidemiological Studies by the Council for International Organizations of Medical Sciences and the World Health Organization (2009), IRB at the college of health sciences, Addis Ababa University, determined that this activity was not human subjects research and its primary intent was public health practice and a disease control activity. Verbal informed consent before examination was obtained from each participant who received a clinical evaluation in Addis Ababa. The purpose of the investigation was explained to all participants. Participants were also informed that their involvement was entirely voluntary and their refusal to answer any question would not result in any negative consequences. To protect participants' confidentiality, personal information was de-identified during data analysis.

Authors' addresses: Wondwossen Amogne, Addis Ababa University, College of Health Sciences, Internal Medicine, Addis Ababa, Ethiopia, E-mail: wonamogne@yahoo.com. Meskerem Nimani and Ishmael Shemsedin, Addis Ababa Emergency Burn \& Trauma Hospital, Emergency Medicine, Addis Ababa, Ethiopia, E-mails: nimamesk@ gmail.com and ishmael.shemsedin@sphmmc.edu.et. Wadu Marshalo, Addis Ababa University, College of Health Sciences, School of Public Health, Addis Ababa, Ethiopia, E-mail: marshwadu2013@gmail.com. Daddi Jima, Ethiopian Public Health Institute, Epidemiology, Addis Ababa, Ethiopia, E-mail: daddi_jima@ yahoo.com. Adamu Addissie, Addis Ababa University, College of Health Sciences, Department of Epidemiology, School of Public Health, Addis Ababa, Ethiopia, E-mail: adamuaddissie@gmail.com. 
John Fogarty, Family \& Community Medicine, University of New Mexico, Albuquerque, NM, E-mail: johnfogarty@me.com.

\section{REFERENCES}

1. Magiorkinis E, Beloukas A, Diamantis A, 2011. Scurvy: past, present, and future. Eur J Intern Med 22: 147-152.

2. WHO, 1999. Scurvy and Its Prevention and Control in Major Emergencies 1999. Available at: who.int/nutrition/publications/ emergencies/WHO_NHD_99.11/en/. Accessed September 23, 2020.

3. Ortner DJ, Butler W, Cafarella J, Milligan L, 2001. Evidence of probable scurvy in subadults from archeological sites in North America. Am J Phys Anthropol 114: 343-351.

4. Paladin A, Wahl J, Zinc A, 2018. Evidence of probable subadult scurvy in the Early Medieval cemetery of Catel Tirolo, South Tyrol, Italy. Int J Osteoarchaeol 28: 714-726.

5. Pitre M, Stark R, Gatto M, 2016. First probable case of scurvy in ancient Egypt at Nag el-Qarmila, Aswan. Int J Paleopathol 13: 11-19.

6. Halcrow SE et al., 2014. First bioarchaeological evidence of probable scurvy in Southeast Asia: Multifactorial etiologies of vitamin $\mathrm{C}$ deficiency in a tropical environment. Int $\mathrm{J}$ Paleopathol 5: 63-71.

7. Padayatty SJ, Levine M, 2016. Vitamin C: the known and the unknown and Goldilocks. Oral Dis 22: 463-493.

8. Hodges RE et al., 1971. Clinical manifestations of ascorbic acid deficiency in man. Am J Clin Nutr 24: 432-443.

9. Burns JJ, 1957. Missing step in man, monkey and guinea pig required for the biosynthesis of L-ascorbic acid. Nature 180: 553.

10. WHO and FAO, 1997. Vitamin and Mineral Requirements in HumanN: Report of a Joint FAO/WHO Expert Consultation. Geneva, Switzerland: World Health Organization.

11. Lamb J, 2016. Scurvy: The Disease of Discovery. Princeton, NJ: Princeton University Press.

12. Bollet AJ, 1992. Scurvy and chronic diarrhea in Civil War troops: were they both nutritional deficiency syndromes? J Hist Med Allied Sci 47: 49-67.

13. Carpenter KJ, 2012. The discovery of vitamin C. Ann Nutr Metab 61: 259-264.

14. Milne I, Chalmers I, 2004. Documenting the evidence: the case of scurvy. Bull World Health Organ 82: 791-796.

15. Szent-Györgyi A, 1928. Observations on the function of peroxidase systems and the chemistry of the adrenal cortex: description of a new carbohydrate derivative. Biochem $J$ 22: 1387-1409.

16. Levavasseur M et al., 2015. Severe scurvy: an underestimated disease. Eur J Clin Nutr 69: 1076-1077.

17. Branquinho DF, et al., 2015. From past sailors' eras to the present day: scurvy as a surprising manifestation of an uncommon gastrointestinal disease. BMJ Case Rep 2015: bcr2015210744.
18. Agarwal A et al., 2015. Scurvy in pediatric age group: a disease often forgotten? J Clin Orthop Trauma 6: 101-107.

19. Gabb G, Gabb B, 2015. Scurvy not rare. Aust Fam Physician 44: 438-440.

20. Velandia B et al., 2008. Scurvy is still present in developed countries. J Gen Intern Med 23: 1281-1284.

21. Desenclos JC et al., 1989. Epidemiological patterns of scurvy among Ethiopian refugees. Bull World Health Organ 67: 309-316.

22. Halestrap P, Scheenstra S, 2018. Outbreak of scurvy in Tana River County, Kenya: a case report. Afr J Prim Health Care Fam Med 10: e1-e3.

23. Hafez $\mathrm{D}$ et al., 2016. Clinical problem-solving: a deficient diagnosis. N Engl J Med 374: 1369-1374.

24. Domingo AE et al., 2016. Now you see/C it. Postgrad Med 128: 629-631.

25. Cheung $E$ et al., 2003. An epidemic of scurvy in Afghanistan: assessment and response. Food Nutr Bull 24: 247-255.

26. CNN, 2002. Congo Fever Fears in Afghanistan. CNN. com, March 12, 2002. Available at: http://www.cnn.com>2002>WORLD $>$ asiapcf $>$ central. Accessed October 20, 2019.

27. Giday A, 2012. Outbreak of scurvy among prisoners in South Ethiopia. Ethiop J Health Dev 26: 60-62.

28. Hodges RE et al., 1969. Experimental scurvy in man. Am J Clin Nutr 22: 535-548.

29. Schorah CJ, 1981. The level of vitamin C reserves required in man: towards a solution to the controversy. Proc Nutr Soc 40: 147-154

30. Watson W, 1956. An outbreak of either scurvy or possibly tropical phlebitis. Cent Afr J Med 2: 365-366.

31. Singh $\mathrm{S}$ et al., 2015. An underdiagnosed ailment: scurvy in a tertiary care academic center. Am J Med Sci 349: 372-373.

32. Pailhous $S$ et al., 2015. Scurvy, an old disease still in the news: two case reports. Arch Pediatr 22: 63-65.

33. Lessing JN et al., 2015. Perifollicular haemorrhage with corkscrew hair due to scurvy. Postgrad Med J 91: 719-720.

34. Fain O, 2005. Musculoskeletal manifestations of scurvy. Joint Bone Spine 72: 124-128.

35. Hirschmann JV, Raugi GJ, 1999. Adult scurvy. J Am Acad Dermatol 41: 895-906, quiz 907-910.

36. Smith A, Di Primio G, Humphrey-Murto S, 2011. Scurvy in the developed world. CMAJ 183: E752-E755.

37. Marshall R, 2002. Oral lesions in scurvy. Aust Dent J 47: 82-83.

38. Packer L, Fuchs J, 1997. Vitamin C in Health and Disease. New York, NY: Marcel Dekker, Inc.

39. Weise Prinzo Z, de Benoist B, 2002. Meeting the challenges of micronutrient deficiencies in emergency-affected populations. Proc Nutr Soc 61: 251-257.

40. Ververs M et al., 2019. Scurvy outbreak among South Sudanese adolescents and young men - Kakuma Refugee Camp, Kenya, 2017-2018. MMWR Morb Mortal Wkly Rep 68: 72-75. 